\title{
Handling of the Grievances in the National Reconstruction Authority (NRA) after the 2015 Earthquake in Nepal
}

\author{
Hikmat Raj Joshi \\ Institute of Engineering, Tribhuvan University, Kathmandu, Nepal \\ Corresponding author: hjoshi@ioe.edu.np
}

Received: March 11, 2019

Revised: May 2, 2019

Accepted: May 5, 2019

\begin{abstract}
An algorithm to handle the grievances from the earthquake victims after the first processing of the data collected from them have been proposed and implemented. Suggestions to improve the performances in data collection, processing and handling have been proposed.
\end{abstract}

Keywords: Grievances, building damage assessment, technical recommendation, earthquake victims, beneficiaries, retrofitting, non-beneficiaries

\section{Introduction}

Handling of the grievances in the NRA after the 2015 earthquake is based upon the data and information surveyed and collected from the households, families in the areas struck by the earthquake and the related people and the authorities. The data and information were collected by authorized people following specific rules, formats and questionnaires. The formats were divided in four sections:

1. Ownership and socioeconomic information

2. Description of the house

3. Photos

4. Grading, recommendations and remarks

Grievances are lodged by the beneficiaries, following the required rules and formalities, when they have disagreements with the decisions made to the data and information collected from them [2]. The objective of this paper is to emphasize the importance of the data and information collected and to improve the procedure of their collection and their assessment for their correctness and usefulness.

\section{Procedure Followed}

The whole procedure was subdivided into three sectors:

i. Data collection and assessment at the site

ii. Data processing in the office

iii. Reassessing, confirming, improving, changing the recommendations or sending for resurvey, if necessary. 
The first survey of the households after the Earthquake was started in March 2016. It included rapid assessment survey of fourteen severely affected districts, Katmandu and seventeen other less affected districts. After the first survey several people lodged their grievances as missed ones or incorrectly assessed. As per the grievances Resurvey/ Reverification (RS/RV) was started in august 2017. In Phase I, the grievances from fourteen severely affected districts were handled and the rest were done in Phase II. The collected data were in prescribed format and at first a first screening was done by the software as per recommendations, damage gradings provided and other conditions like HIOP/ HIUP (House in Other/ Usual Place). They were classified into three groups:

i. Reconstruction Beneficiaries (RCB)

ii. Retrofitting Beneficiaries (RTB)

iii. Non-Beneficiary (NB)

The first two groups were supposed to receive $R s 300 k$ and $R s 100 k$ respectively. The screening results were found to be beyond the realization of the NRA and also there were a lot of grievances lodged for reconsideration of the results from the screening. The number of grievances were some 129,410 and 122,000 for Phase I and Phase II, respectively. A decision was made by the NRA to review the screening results by the Engineers from NRA. It was organized to be done by two teams of Engineers. The first team (Team I) consisted of 25 Engineers and each of them were tasked to go through and deliberate on the data, information, photos, remarks, damage gradings and recommendations. The next thing they were tasked to do was to provide recommendation with additional remarks, if needed. The last remarks were to be put after a space and slash (_/) to distinguish it from the earlier one. The recommendations they make are based upon the data provided and the agreed set of rules as shown in the Fig. 1. As per data and information from the assessment matrix, photo and socioeconomic data the recommendation could be Reconstruction (RCB), Retrofitting (RTB), or Non (NB) Beneficiaries. The recommendations are made according to the damage level, as prescribed in international standards $[1,4]$ assessed by the Engineer based upon the data and information as shown in Fig. 2. The recommendation could be with or without conditions clamped to it, depending upon the information available or unavailable and other social conditions like extended family, HIOP etc. For the conditional recommendations the conditions would be and/or procedures to be followed are explained in the remarks. The Recommendation for Reconstruction Beneficiaries (RCB), depending upon conditions are attached or not would be Potential (PTB) or Probable (PRB) respectively. Similarly, the Recommendations for Retrofitting Beneficiaries (RTB) could be with or without conditions attached to them depending upon whether some conditions are to be fulfilled before the implementation of the Recommendation or not. Each Engineer would start with grievances of a certain locality (urban/village municipality), finish it and go for the next one.

In this process, if the Engineers come across challenges like:

i. Not enough data/information

ii. The structure of the house or the conditions are too complicated to make decision or recommendation for them,

They just mention it in the 'Remarks' and put 'Others' as the recommendation. All the grievances with 'Others' in Recommendation from the Team I Engineers are passed to the Team II Engineers. The Team II Engineers comprises three to five Senior Engineers or selectively trained Engineers to work with the Senior Engineers. They once again go through the cases with 'Others' in the 
Recommendation from the Team I and look into the information (matrix, photos and socio economic data), reason and think in a broader and higher plane and try to supply 'Recommendation' as prescribed in Figure 2 with reasons and conditions explained in the 'Remarks'. If the Team II cannot solve the case in this way, they pass the case to MIS team as it could be with wrong/incomplete/ mismatched data/information, for redoing it or for resurvey. They are termed as Defective Data. The Defective Data are classified into three groups: wrongly entered-'Error', wrongly processed -'unupdatable' and showing no record in the file -'DNF' (Data Not Found)

\begin{tabular}{|c|c|}
\hline $\begin{array}{c}\text { Error: } \\
\text { Unupdatable: }\end{array}$ & Shows 'Error' when referred \\
\hline DNF: & Cannot be updated \\
\hline
\end{tabular}

\section{Suggestions for the Next Phase}

\subsection{Survey}

a. Matrix

- All of the elements of the matrix should be filled in or explained as Not Applicable (NA) or Unavailable (UA)

- Each entry should be directed and targeted correctly to description and grading of the house

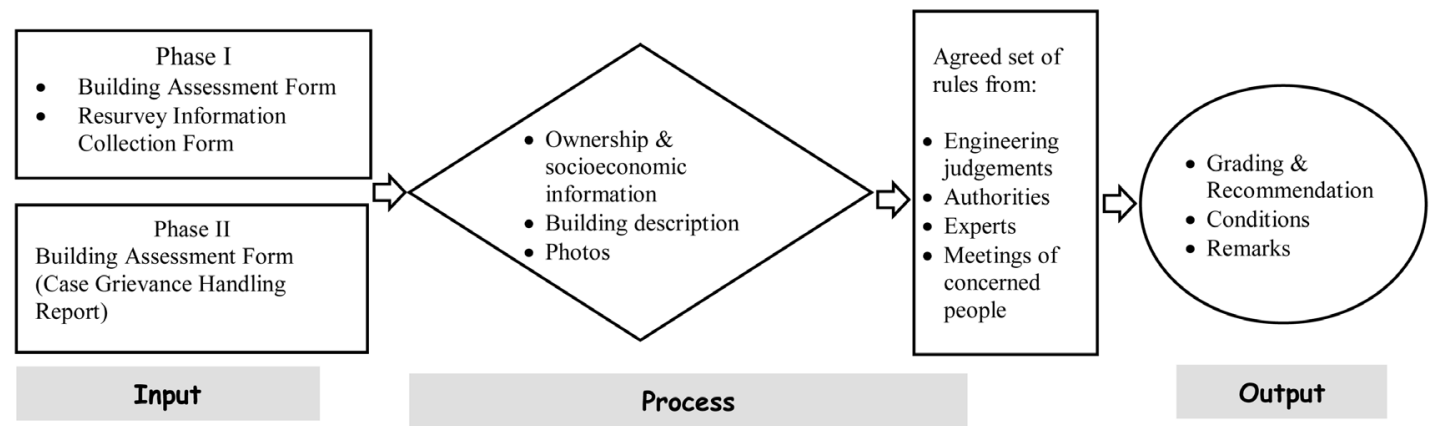

Fig. 1: Decisioning Flow chart

- Any deviation, mismatch, lapse or absence of data/information should be explained in the remarks.

- The General Comments must be given as overall observation and should not be left blank.

\section{b. Photos}

- The photos should be snapped and supplied to clearly describe:

i. Volume, shape and size of the house (4 elevations +1 with other houses in background, if available).

ii. System (structural system like frame, wall, Roof etc.)

iii. Materials (mud, concrete, timber etc.) 
iv. Damages (local-cracks, spalling, yieldings etc; collapses-partial, total etc), separately for inner and outer, local and global.

v. Photos should be labeled and captioned to explain clearly what is the objective of the photo.

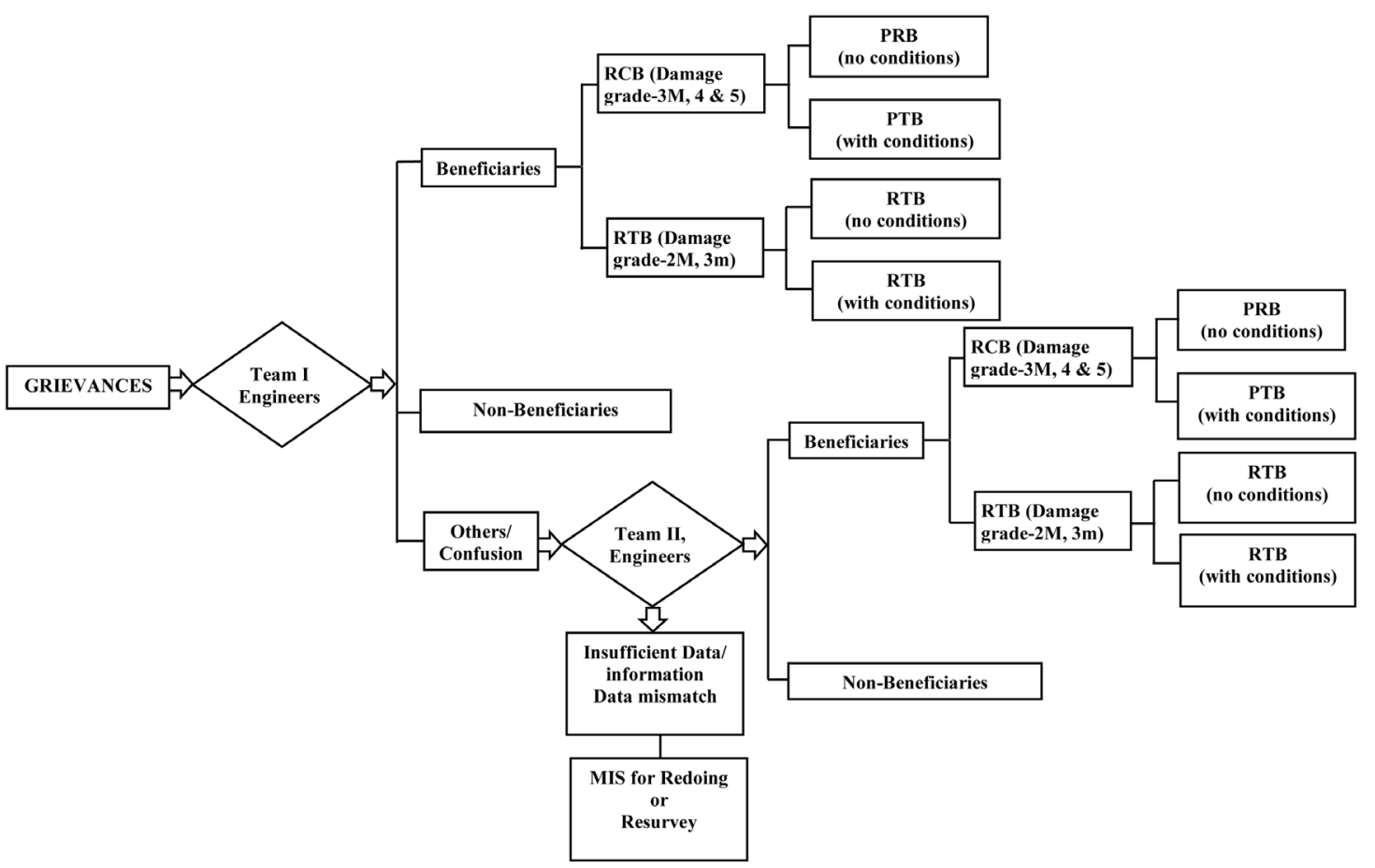

Fig. 2: Recommendation Flow Chart

\section{c. Socioeconomic data}

- All of the elements of the matrix should be filled in or explained as Not Applicable (NA) or Unavailable (UA).

- Each entry should be directed and targeted correctly to description and grading of the family, its volume, socioeconomic status, number of houses owned (HIOP, HIUP) etc.

- Any deviation, mismatch, lapse or absence of data/information should be explained in the remarks.

\section{d. Assessment and Recommendation}

- The Remarks should briefly and clearly explain how the damage assessment was done, if it is not clear from the matrices and photos.

- The damage assessment and the Recommendation must be in agreement with the standards and norms [1, 4, Figure 2].

- The Conditional Recommendations should necessarily accompany the explanations of the conditions clamped, in the remarks.

- Any of the earlier 'Remarks' from the site, Team I or Team II must not be deleted but separated by a space and a slash (_/) and continued. 


\subsection{Team I Engineers}

- Choose the team leader and conduct regular meetings (at least once in a week for an hour) to maintain the uniformity in quality and quantity of the works done. (eg. the assessments made for SMM and thatch roofed houses)

- Attend/conduct regular meetings with Team II Engineers, concerned authorities and representatives from MIS and administrative team at least once in a month or as per requirements to discuss the challenges and to maintain common language and uniformity in handling of the grievances.

- Maintain the records and circulate the decisions made in the meetings for handling of the grievances.

\subsection{Team II Engineers}

- Plan and develop a separate chain of commands among the Engineers and other authorities for handling the grievances.

- Develop rules, policies and procedures to handle grievances.

For instance, some of the considerations done and followed as rules were as follows:

i. Proximity to the epicenter (10 $\mathrm{km}$ from Barpak or Sunkhani)

ii. Brick/stone in mud mortar houses (B/SMM houses)

iii. Thatch roofed houses

iv. Hybrid houses (ground floor SMM and upper floor timber)

v. Timber houses

vi. Ramshackle and makeshift houses

vii. Big families (more than 5 members) and very big families (more than 8 members)

viii. Elderly people as separate and independent member of the family, when they request for it

ix. Access for appeal for reconsideration

x. Vulnerability of the houses to the earthquake

- Conduct regular meetings with the Team I Engineers, concerned authorities and MIS people to propagate rules and policies in handling grievances.

- Do the documentation, maintain the record of the rules and policies developed and time line of performances of the Engineers to achieve the target set.

\section{Conclusions and Recommendations}

- An algorithm of surveying and recording of data/information pertaining to the state of the houses and status of the family with respect to the socioeconomic aspects, after an earthquake has been developed.

- A procedure to handle these data to recommend mitigation and preventive measures has been designed.

- A tool to handle the grievances or complaints from the concerned people has been prepared and used.

- Adopting the described algorithm, procedure and tool, 122,070 and 101,275 grievances, respectively from Phase I and Phase II have been handled successfully. Only 59 grievances 
from Phase II are unsolved due to insufficiency/mismatch of data/information and they have been sent to the MIS section for redoing.

- It is suggested that the algorithm, procedure and tool worked out and implemented could be generalized and tailored for other cases as well.

- The suggestions for improvement in survey and other steps of the procedure are targeted to mitigate the challenges and obstacles faced in Phase I and Phase II.

Acknowledgement: The author would like to acknowledge to Dr. Hari Ram Parajuli, Er Nabin Paudel, Er Megha Raj Sharma, Er Smriti Rajbhandari, Er Julisha Pradhan, Er Pragya Subedi, Er Sunita Kutu and Er Anju Khadka for their cooperation to prepare this paper.

\section{References}

[1] Classification of Damage according to EMS98.

[2] Grievances Management Related to Reconstruction and Rehabilitation Guidelines (2018 A D 2073 B S), Unofficial Translation conducted by HRRP and partner organization.

[3] https://ehrpmis.nra.gov.np.

[4] Okada S and Takai N (1999), Classifications of structural types and damage patterns of buildings for earthquake field, Journal of structural and Construction Engineering (Transactions of AIJ) 525(524), DOI:10.3130/aijs.64.65_5.

[5] Reconstruction related laws, rules, norms and directives (2018), Government of Nepal, National Reconstruction Authority. 